\title{
HIGHLY MULTIPLICATIVE SERIES
}

This paper was downloaded from TechRxiv (https://www.techrxiv.org).

\section{LICENSE}

CC BY 4.0

SUBMISSION DATE / POSTED DATE

05-02-2022 / 22-02-2022

CITATION

Pandey, Vishal (2022): HIGHLY MULTIPLICATIVE SERIES. TechRxiv. Preprint. https://doi.org/10.36227/techrxiv.19126634.v1

$\mathrm{DOI}$

10.36227/techrxiv.19126634.v1 


\title{
HIGHLY MULTIPLICATIVE SERIES
}

\author{
Author Name: Vishal Pandey ${ }^{1}$ \\ ${ }^{12}$ St Thomas' College of Engineering and Technology \\ ${ }^{12}$ Kolkata, India
}

\begin{abstract}
In the Fibonacci series we have two numbers by adding them we get a series consisting of even and odd numbers in this it goes up to infinity we can track any $\mathbf{n}^{\text {th }}$ number by the Binet's formula. I have just thought of the multiplication of the first two terms and continued till where I can go, it means that the first two terms in the form ( $a, b)$ we will continue the multiplication as we do the addition in the Fibonacci series. As a result we will get the big integers from the $7^{\text {th }}$ term approximately which is obvious by multiplying to its previous one it will come to an a very big integer which cannot be accountable by some range. If we do the multiplication the first two terms will be the same however from the third term it can be written as the power of that integers in which the powers will be following the Fibonacci series in this we can also find the $\mathbf{n}^{\text {th }}$ term for the multiplicative series. Here the first two terms will in the same order as it will be given to find the series by changing the order it will violates the rule of restricted term. The meaning of the restricted here is that the order of $(a, b)$ will be the same throughout the calculation of whole series we cannot alter that if we do so them it will not be more restricted term. So there are two concept in the multiplicative series restricted and non-restricted series. If the (a, b) is there and the operation is going on then it can be said as the restricted series if it is given $(a, b)$ and asked for the $(b, a)$ series then it is said as non-restricted series. I have considered 4 possible criteria to check the pairing of the variables $(a, b)$. We will get to know about the series and also the $n^{\text {th }}$ term value of that series for all possible solutions.
\end{abstract}

Keyword: mathematics, algebra, series.

\section{INTRODUCTION}

We have learned about the basics of Fibonacci series in which the series is being of addition of previous two terms and keep going on until you want. If there is an unknown term and we want the value from the series then we will find it from the Binet formula.

From the think if we do the multiplication of two previous terms as like Fibonacci series then we will get different results and also we have to find the $\mathrm{n}^{\text {th }}$ term in a different way. 
In the Fibonacci series all the major role is the first and the second term and also the addition. Here I have think about if the 5 different combinations of first and second terms which results in highly multiplicative numbers and combinational of the numbers are called highly multiplicative series.

\section{THEORY}

Highly multiplicative series means that the series in which the first and the second terms are get multiplied till up to infinity and the power of the two consecutive first and second term should follow the Fibonacci series.

From Fibonacci series, we know: 1,1,2,3,5,8,13,21,34,55,89,144,233,... and so on.....(i)

We have the first and second terms are:

first term $=1$ and second term $=1$, and then we add them to the previous result which comes up to a series.

Now, I thought for the multiplication if the first term should get multiplied then what would be the result??

From the above series we have:- $1,1,1,1,1, \ldots \ldots \ldots$ and so on (because here the first and second terms are equal to 1 )

If we think that the first and second terms are denoted as the (a, b) and we proceed further as the multiplication. How we can go??

$(a, b)=(1,2)=1,2,2,4,8,32,256,8192,2097152,1.71799 \times 10^{10}, \ldots \ldots .$. and so on $\ldots(i i)$

Here we how make a series which get higher and higher until we stop this.

Here we make the above series $(i i)$ :-

first term:- 1

second term:- 2

third term:- $2 \mathrm{X} 1=2$

fourth term:- (third term) $\mathrm{X}$ (second term) $=2 \mathrm{X} 2=4$

fifth term:- (fourth term) $\mathrm{X}$ (third term) $=4 \mathrm{X} 2=8$

sixth term:- (fifth term) $\mathrm{X}$ (fourth term) $=8 \mathrm{X} 4=32 \ldots \ldots \ldots$ and so on...

Here the first and second terms are can be of any integer so there are 4 probabilities to for these terms.

The 4 conditions are:-

1. If $a=1$ and $b=$ any random integer

eg: $a=1$ and $b=2$ or 3 or 4 or 5 or 6 or 7 or $8, \ldots \ldots$ or any other integers 
2. If $a=$ any random integer and $b=1$

eg:- $b=1$ and $a=2$ or 3 or 4 or 5 or 6 or 7 or $8, \ldots \ldots$ or any other integers

3 . If $a=$ any random integer $\left(n_{1}\right)$ and $b=$ any random integer $\left(n_{2}\right)$

eg:- $a=1$ or 2 or 3 or 4 or 5 or 6 or 7 or $8, \ldots \ldots$ or any other integers and $b=$ 1 or 2 or 3 or 4 or 5 or 6 or 7 or $8, \ldots \ldots .$. or any other integers

4. If $a=b$

eg;- $(a, b=1,1)$ or $(a, b=2,2)$ or $(a, b=3,3)$ or $(a, b=$ $99,99)$ or any other integers

Now we will make the series and also check for the Fibonacci series in the power from these all four conditions.

\section{If $a=1$ and $b=$ any random integer}

Eg:- $(a, b)=(1,2)$ Let us suppose the first two terms be 1 and 2 and we will check for the series

$$
(a, b)=(1,2)=1,2,2,4,8,32,256,8192,2097152,1.71799 \times 10^{10}, \ldots \ldots .(i i i)
$$

first term:- 1

second term:- 2

third term:- $2 \mathrm{X} 1=2$

fourth term:- (third term) $\mathrm{X}$ (second term) $=2 \mathrm{X} 2=4$

fifth term:- (fourth term) $\mathrm{X}$ (third term) $=4 \mathrm{X} 2=8$

sixth term:- (fifth term) $\mathrm{X}$ (fourth term) $=8 \mathrm{X} 4=32 \ldots \ldots \ldots$ and so on...

Now we know from the first term and second term lets go from the third term...

From (iii)

$(a, b)=(1,2)=2^{1}, 2^{2}, 2^{3}, 2^{5}, 2^{8}, 2^{13}, 2^{21}, \ldots \ldots \ldots . .$. and so on from the third term $\}$

Now here we look carefully we can see the powers are in the Fibonacci series.

We can see from the other examples such as the,

$(1,3),(1,4),(1,5), \ldots . .(1,99), \ldots$ and so on

Eg:- $(a, b)=(1,3)=1,3,3,9,27,243,6561,1594323,1.04603532 \times 10^{10}, 1.66771817 \times 10^{16}$, and so on $\ldots$

Now we know from the first term and second term lets go from the third term...

$$
(a, b)=(1,3)=3^{1}, 3^{2}, 3^{3}, 3^{5}, 3^{8}, 3^{13}, 3^{21}, 3^{34}, \ldots \ldots . \text { and so on }
$$

As we know the Fibonacci series is:- $1,1,2,3,5,8,13,21,34,55,89,144,233, \ldots \ldots$. 
From above series, the $8^{\text {th }}$ term of the Fibonacci is 21 .

The highly multiplicative series of $(1,2)$ is:- $1,2,2^{1}, 2^{2}, 2^{3}, 2^{5}, 2^{8}, 2^{13}, 2^{21}, \ldots \ldots \ldots$ From the above series of $(1,2)$ we can assign the terms as,

first term $=1$

second term $=2$

third term $=2^{1}$

fourth term $=2^{2}$

fifth term $=2^{3}$

sixth term $=2^{5}$

seventh term $=2^{8}$

eight term $=2^{13}$

ninth term $=2^{21} \ldots \ldots$...and so on...

If we want to find the $\mathrm{n}^{\text {th }}$ term from the Fibonacci series to the multiplicative series then it should match the integer which we would get i.e. the $\mathrm{n}^{\text {th }}$ term value of the Fibonacci series should be equal to the power raised by the $(a, b)$.

The $(\boldsymbol{n})^{\text {th }}$ term of Fibonacci series is equal to the $(n+1)^{\text {th }}$ term of the highly multiplicative series.

So that's why for given in question for the $\mathrm{n}^{\text {th }}$ term of the highly multiplicative series we should find the $(\mathrm{n}-1)^{\text {th }}$ term of the Fibonacci series or sequence and then we can proceed for further calculation.

If we want to find the $9^{\text {th }}$ term of the multiplicative series of $(1,2)$ with respect to the Fibonacci series?

Solution:- $\mathrm{n}=9$ so for Fibonacci we should $\mathrm{n}-1=8$

The $8^{\text {th }}$ term form the Fibonacci series is 21 then it should match the power of $(1,2)$ in series.

So, in above series the ninth $\left(9^{\text {th }}\right)$ term of multiplicative series has the power of 21 and it is the $8^{\text {th }}$ term of the Fibonacci series.

So the $8^{\text {th }}$ term of the multiplicative series of $(1,2)$ w.r.t the Fibonacci series is $=2^{21}$

Some more questions randomly for checking it.......

If we want to find the $6^{\text {th }}$ term of the multiplicative series of $(1,3)$ with respect to the Fibonacci series?

Solution:- $\mathrm{n}=6$ so for Fibonacci we should $\mathrm{n}-1=5$ 
The $5^{\text {th }}$ term form the Fibonacci series is 5 then it should match the power of $(1,3)$ in series.

$$
(1,3)=1,3,3^{1}, 3^{2}, 3^{3}, 3^{5}, 3^{8}, 3^{13}, 3^{21}, 3^{34}, \ldots \ldots \ldots
$$

So, in above series the $\operatorname{sixth}\left(6^{\text {th }}\right)$ term of multiplicative series has the power of 21 and it is the $5^{\text {th }}$ term of the Fibonacci series.

So the $5^{\text {th }}$ term of the multiplicative series of $(1,3)$ w.r.t the Fibonacci series is $=2^{5}=32$

Therefore we can see that the $n^{\text {th }}$ term of the Fibonacci series is equal to the $(n+1)^{\text {th }}$ term of the highly multiplicative series.

For higher numbers:-

If we want to find the $8^{\text {th }}$ term of the multiplicative series of $(1,99)$ with respect to the Fibonacci series?

Solution: $\mathrm{n}=8$ so for Fibonacci we should $\mathrm{n}-1=7$

The $7^{\text {th }}$ term form the Fibonacci series is 13 then it should match the power of $(1,99)$ in series.

$$
(1,99)=1,99,99^{1}, 99^{2}, 99^{3}, 99^{5}, 99^{8}, 99^{13}, 99^{21}, 99^{34},
$$

So, in above series the eight $\left(8^{\text {th }}\right)$ term of multiplicative series has the power of 13 and it is the $7^{\text {th }}$ (seventh) term of the Fibonacci series.

So the $8^{\text {th }}$ term of the multiplicative series of $(1,99)$ w.r.t the Fibonacci series is $=99^{13}=8.77521023 \times 10^{25}$.

This was finding the term w.r.t the Fibonacci number. If it is written without any w.r.t the Fibonacci series then follow the standard process of finding the term and start the process.

If you want to know the $30^{\text {th }}$ or $60^{\text {th }}$ or $75^{\text {th }}$ term of the Fibonacci then it can be get by the Binet Formula.

The Binet formula is : $\frac{(1+\sqrt{5})^{n}-(1-\sqrt{5})^{n}}{2^{n} \sqrt{5}}$

So if we want to know higher term of the Fibonacci then we use this formula and after getting the value of that we should go for finding the highly multiplicative series.

Eg:- If we want to find the $31^{\text {st }}$ term of the multiplicative series of (1, 99) with respect to the Fibonacci series?

Solution:- $\mathrm{n}=31$ so for Fibonacci we should n-1 $=30$

From Binet formula:- $\frac{(1+\sqrt{5})^{n}-(1-\sqrt{5})^{n}}{2^{n} \sqrt{5}}$ [by putting $\mathrm{n}=30$ ] 


$$
\begin{aligned}
& \frac{(1+\sqrt{5})^{30}-(1-\sqrt{5})^{30}}{2^{30} \sqrt{5}}=\frac{\left(1.997694516 \times 10^{15}\right)-(577.1260297)}{(1073741824) X(2.236067977)}=\frac{\left(1.997694516 \times 10^{15}\right)}{2400959709}= \\
& 832039.9999 \approx 832040 \text { (approx) }
\end{aligned}
$$

Getting the $30^{\text {th }}$ term from the Fibonacci series it means that the highly multiplicative series should contain the Fibonacci term at the power in $31^{\text {st }}$ term.

So the $31^{\text {st }}$ term of highly multiplicative series of $(1,99)$ is : $99^{832040}$ (which is very high number and this is the correct following term if we proceed).

In general,

- $(a, b)=(1, n)=1, n, n^{1}, n^{2}, n^{3}, n^{5}, n^{8}, n^{13}, n^{21}, n^{34}, n^{55}, \ldots \ldots . . e t c$ \{restricted or highly multiplicative series\}

- For higher term, we would use $\frac{(1+\sqrt{5})^{n}-(1-\sqrt{5})^{n}}{2^{n} \sqrt{5}}$ to get the term from the Fibonacci series and then we can find the $n^{\text {th }}$ term of the highly multiplicative series.

- The $(n)^{\text {th }}$ term of Fibonacci series is equal to the $(n+1)^{\text {th }}$ term of the highly multiplicative series.So that's why for given in question for the $\mathrm{n}^{\text {th }}$ term of the highly multiplicative series we should find the $(n-1)^{\text {th }}$ term of the Fibonacci series or sequence and then we can proceed for further calculation.

\section{If $a=$ any random integer and $b=1$}

Eg:- $(a, b)=(2,1)$ Let us suppose the first two terms be 2 and 1 and we will check for the series

$(a, b)=(1,2)=2,1,2,4,8,32,256,8192,2097152,1.71799 x 10^{10}, \ldots \ldots$

first term:- 2

second term:- 1

third term:- $2 \mathrm{X} 1=2$

fourth term:- (third term) $\mathrm{X}$ (second term) $=2 \mathrm{X} 2=4$

fifth term:- (fourth term) X (third term) $=4 \mathrm{X} 2=8$

sixth term:- (fifth term) X (fourth term) $=8 \mathrm{X} 4=32 \ldots \ldots \ldots$ and so on. .

Now we know from the first term and second term lets go from the third term...

From (iii)

$(a, b)=(1,2)=2^{1}, 2^{2}, 2^{3}, 2^{5}, 2^{8}, 2^{13}, 2^{21}$, ..and so on $\{$ from the third term $\}$

Now here we look carefully we can see the powers are in the Fibonacci series.

We can see from the other examples such as the,

$(3,1),(4,1),(5,1), \ldots . .(99,1), \ldots$ and so on 
Eg:- $(a, b)=(3,1)=3,1,3,9,27,243,6561,1594323,1.04603532 X 10^{10}, 1.66771817 X 10^{16}$, and so on ...

Now we know from the first term and second term lets go from the third term...

$$
(a, b)=(3,1)=3^{1}, 3^{2}, 3^{3}, 3^{5}, 3^{8}, 3^{13}, 3^{21}, 3^{34}, \ldots \ldots \text { and so on }
$$

The highly multiplicative series of $(1,2)$ is:- $2,1,2^{1}, 2^{2}, 2^{3}, 2^{5}, 2^{8}, 2^{13}, 2^{21}, \ldots \ldots$. From the above series of $(1,2)$ we can assign the terms as,

first term $=2$

second term $=1$

third term $=2^{1}$

fourth term $=2^{2}$

fifth term $=2^{3}$

sixth term $=2^{5}$

seventh term $=2^{8}$

eight term $=2^{13}$

ninth term $=2^{21} \ldots \ldots$.. and so on. .

If we want to find the $\mathrm{n}^{\text {th }}$ term from the Fibonacci series to the multiplicative series then it should match the integer which we would get i.e. the $\mathrm{n}^{\text {th }}$ term value of the Fibonacci series should be equal to the power raised by the $(a, b)$.

The $(\boldsymbol{n})^{\text {th }}$ term of Fibonacci series is equal to the $(n+1)^{\text {th }}$ term of the highly multiplicative series.

So that's why for given in question for the $\mathrm{n}^{\text {th }}$ term of the highly multiplicative series we should find the $(n-1)^{\text {th }}$ term of the Fibonacci series or sequence and then we can proceed for further calculation.

Every calculation will be same as just we have did before in $(a, b)=(1, n)$

The $n^{\text {th }}$ term of the highly composite series is equal to the $(n-1)^{\text {th }}$ term of the Fibonacci series

In general,

- $(a, b)=(n, 1)=n, 1, n^{1}, n^{2}, n^{3}, n^{5}, n^{8}, n^{13}, n^{21}, n^{34}, n^{55}, \ldots \ldots . . e t c$ (nonrestricted or reversible highly multiplicative series)

- For higher term, we would use $\frac{(1+\sqrt{5})^{n}-(1-\sqrt{5})^{n}}{2^{n} \sqrt{5}}$ to get the term from the Fibonacci series and then we can find the $n^{\text {th }}$ term of the highly multiplicative series. 
- The $(n)^{t h}$ term of Fibonacci series is equal to the $(n+1)^{\text {th }}$ term of the highly multiplicative series.So that's why for given in question for the $\mathrm{n}^{\text {th }}$ term of the highly multiplicative series we should find the $(n-1)^{\text {th }}$ term of the Fibonacci series or sequence and then we can proceed for further calculation.

\section{If $\mathbf{a}=\mathbf{b}$}

In this the $\mathrm{a}$ and $\mathrm{b}$ will be equal as example: $(1,1),(2,2)$, $(3,3), \ldots \ldots \ldots(99,99), \ldots \ldots \ldots(1987,1987), \ldots \ldots(20000001,20000001), \ldots \ldots \ldots$ etc

As both number is equal it will be easier to get compare the term from the Fibonacci series.

$(a, b)=(2,2)=2,2,4,8,32,256,8192,2097152,1.717986918 \times 10^{10}, \ldots \ldots \ldots \ldots$ etc

first term:- 2

second term:- 2

third term:- $2 \mathrm{X} 1=4$

fourth term:- (third term) $\mathrm{X}$ (second term) $=4 \mathrm{X} 2=8$

fifth term:- (fourth term) $\mathrm{X}$ (third term) $=8 \mathrm{X} 4=32$

sixth term:- $($ fifth term) $\mathrm{X}$ (fourth term) $=8 \mathrm{X} 32=256 \ldots \ldots$ and so on...

We can say,

$$
\begin{aligned}
& (a, b)=(2,2)=2^{1}, 2^{1}, 2^{2}, 2^{3}, 2^{5}, 2^{8}, 2^{13}, \ldots \ldots \text { etc } \\
& (a, b)=(3,3)=3^{1}, 3^{1}, 3^{2}, 3^{3}, 3^{5}, 3^{8}, 3^{13}, \ldots \ldots \text { etc } \\
& (a, b)=(4,4)=4^{1}, 4^{1}, 4^{2}, 4^{3}, 4^{5}, 4^{8}, 4^{13}, \ldots \ldots \text { etc } \\
& (a, b)=(99,99)=99^{1}, 99^{1}, 99^{2}, 99^{3}, 99^{5}, 99^{8}, 99^{13}, \ldots \ldots \text { etc }
\end{aligned}
$$

Now from Fibonacci series: $1,1,2,3,5,8,13,21,34,55, \ldots$...and so on

Here we can see that the each term of Fibonacci series is equal to the power of the $(a, b)$. 
If we want to find the $6^{\text {th }}$ term of the multiplicative series of $(3,3)$ with respect to the Fibonacci series?

$$
(a, b)=(3,3)=3^{1}, 3^{1}, 3^{2}, 3^{3}, 3^{5}, 3^{8}, 3^{13}, \ldots \ldots \text { etc }
$$

From Fibonacci series we get: $1,1,2,3,5,8,13,21,34,55 \ldots \ldots \ldots$

The $6^{\text {th }}$ of the Fibonacci series is: 5 .

Now from the highly multiplicative series we can get on the $6^{\text {th }}$ term: $3^{5}=243$

In general,

- $(a, b)=(n, n)=\left(n, n, n^{2}, n^{3}, n^{5}, n^{8}, n^{13}, \ldots\right.$.etc $)$

- Here we can easily get the term from the Fibonacci series and can compare of the highly multiplicative series.

- Here the $\mathrm{n}^{\text {th }}$ term of the Fibonacci series is equal to the $\mathrm{n}^{\text {th }}$ term of the highly multiplicative series.

4. If $\mathbf{a}=$ any random $\operatorname{integer}\left(\mathbf{n}_{\mathbf{1}}\right)$ and $\mathbf{b}=$ any random integer $\left(\mathbf{n}_{\mathbf{2}}\right)$

In this the $\mathrm{a}$ and $\mathrm{b}$ will be any number so, as example: $(2,3),(7,2)$

$(2,9), \quad(3,4), \ldots \ldots \ldots(909,949), \ldots \ldots \ldots(198,998), \ldots \ldots(2000$, $20000001), \ldots \ldots$. . etc

$(a, b)=(2,3)=2,3,6,18,108,1944,209952,408146688,8.569121344 \times 10^{13}$ etc

$(a, b)=(2,4)=2,4,8,32,256,8192,2097152,1.7199 X 10^{13} \ldots \ldots \ldots$.etc

$(a, b)=(2,7)=\left(2,7,14,98,1372,134456,184473632,2.48 \times 10^{13}\right.$.

$(a, b)=(3,19)=3,19,57,1083,61731,66854673 \ldots$..etc

We can say:

$(2,3)=2,3^{1}, 2^{1} 3^{1}, 2^{1} 3^{2}, 2^{2} 3^{3}, 2^{3} 3^{5}, 2^{5} 3^{8}, 2^{8} 3^{13}, \ldots \ldots$ and so on $\ldots$

$(2,4)=2,4^{1}, 2^{1} 4^{1}, 2^{1} 4^{2}, 2^{2} 4^{3}, 2^{3} 4^{5}, 2^{5} 4^{8}, \ldots \ldots$. and so on ...

$(2,7)=2,7^{1}, 2^{1} 7^{1}, 2^{1} 7^{2}, 2^{2} 7^{3}, 2^{3} 7^{5}, 2^{5} 7^{8}, \ldots \ldots$ and so on $\ldots$.

$(3,19)=3,19^{1}, 3^{1} 19^{1}, 3^{1} 19^{2}, 3^{2} 19^{3}, 3^{3} 19^{5}, 3^{5} 19^{8}, 3^{8} 19^{13}$ and so on

We can write the terms as follows for $(2,3)$ :

first term: - 2

second term: $-3^{1}$

third term: $-2^{1} 3^{1}$

fourth term: $-2^{1} 3^{2}$ 
fifth term: $-2^{2} 3^{3}$

sixth term: $-2^{3} 3^{5}$

seventh term: $-2^{5} 3^{8} \ldots$ and so on...... $\left(^{*}\right)$

From Fibonacci terms the power of highly multiplicative series should be in an order.

By neglecting the first two terms of highly multiplicative series.

Fibonacci series:- $1,1,2,3,5,8,13,21,34,55,89,144, \ldots \ldots \ldots$ and so on

Fibonacci terms:-

first term: -1

second term: -1

third term: -2

fourth term: - 3

fifth term: -5

sixth term: -8

seventh term: $-13 \ldots \ldots$...and so on

$2^{1} 3^{1}$ : - In Fibonacci series the first two terms are 1,1. So, the sequence of term in the series from the Fibonacci is $(1,2)$.

$2^{1} 3^{2}:$ - sequence-term $=(2,3)$

$2^{2} 3^{3}:$ - sequence-term $=(3,4) \ldots$ and so on

It means in simple way that the if we take powers of highly multiplicative series and make a term sequence then: -

By neglecting the first and second term in highly multiplicative series

Power sequence of highly multiplicative series: - $(1,1),(1,2),(2,3),(3,5)$, $(5,8),(8,13) \ldots$ and so on....

So, from the Fibonacci series the term sequence is: - (first term, second term), (second term, third term), (third term, fourth term), (fourth term, fifth term) .... and so on...

If we write in numbers: - $(1,2),(2,3),(3,4),(4,5) \ldots$ and so on. .

So in general the sequence term is : $(\mathbf{n},(\mathbf{n}+\mathbf{1}))$

Here for each pair of first and second term in highly multiplicative series we get the Fibonacci series in the power of given pair from the third term.

So, we can say that in $(\mathrm{a}, \mathrm{b})$ : $\mathrm{a}$ is the previous Fibonacci term and $\mathrm{b}$ is the next Fibonacci term in the given Fibonacci series. 
Now for getting the respective term in highly multiplicative series from the given term of the Fibonacci series should follow from third term of highly multiplicative series. So, we will neglect the first two terms (i.e., first and second term).

Eg:- If we want to find the $6^{\text {th }}$ term of the multiplicative series of (2,3) with respect to the Fibonacci series?

\section{Solution:-}

$n=6$

as we will neglect the first two terms so, $n=6-2=4$

Sequence term:- $(\mathrm{n},(\mathrm{n}+1))=(4,5)$

Now checking from the Fibonacci series values of term we get:-

Fibonacci series:- $1,1,2,3,5,8,13,21,34,55,89,144, \ldots \ldots \ldots$ and so on

Fibonacci term:-

$4^{\text {th }}$ term:- 3

$5^{\text {th }}$ term:- 5

Now the term pair is :- $(3,5)$.

Here the value of $(\mathrm{a}, \mathrm{b})$ is $(2,3)$ hence the $6^{\text {th }}$ term is:- $2^{3} 3^{5}$

Check from the above series we are correct. Hence the value is correct and we can find any tern of highly multiplicative series of any random number pairs of first and second term.

Eg:- If we want to find the $30^{\text {th }}$ term of the multiplicative series of (2, 3) with respect to the Fibonacci series?

\section{Solution:-}

$\mathrm{n}=30$

as we will neglect the first two terms so, $n=30-2=28$

Sequence term:- $(\mathrm{n},(\mathrm{n}+1))=(28,29)$

We cannot write the Fibonacci series for all the time we can find the term values from the Binet equation: $\frac{(1+\sqrt{5})^{n}-(1-\sqrt{5})^{n}}{2^{n} \sqrt{5}}$

So for $\mathrm{n}=28$ :

$\frac{(1+\sqrt{5})^{28}-(1-\sqrt{5})^{28}}{2^{28} \sqrt{5}}=\frac{\left(1.907628515 \times 10^{14}\right)-(377.7338904)}{600239927.2}=317811$

Now for $\mathrm{n}=29$ : 


$$
\frac{(1+\sqrt{5})^{29}-(1-\sqrt{5})^{29}}{2^{29} \sqrt{5}}=\frac{\left(6.17321555 \times 10^{14}\right)-(-466.9047659)}{1200479854}=514229
$$

Now the term pair is :- $(317811,514229)$.

Here the value of $(a, b)$ is $(2,3)$ hence the $30^{\text {th }}$ term is:- $2^{317811} 3^{514229}$

In general:

- $(a, b)=\left(n_{1}, n_{2}\right)=\left(n_{1}, n_{2}, n_{1}{ }^{1} n_{2}{ }^{1}, n_{1}{ }^{1} n_{2}{ }^{2}, n_{1}{ }^{2} n_{2}{ }^{3}, n_{1}{ }^{3} n_{2}{ }^{5}, n_{1}{ }^{5} n_{2}{ }^{8}, n_{1}{ }^{8} n_{2}{ }^{13} \ldots\right.$. etc $)$

- Neglect the two terms i.e., the first and second term from the series and find the sequence term pair as explained in above, and match them with the Fibonacci values of the series and put the values to the pair $(a, b)$ and get the $\mathrm{n}^{\text {th }}$ term which we want the desired result.

- Here we neglect the 2 terms (first and second terms) because the series follow the Fibonacci series from the third term.

\section{CONCLUSION}

In this theory I have think the Fibonacci series in other terms by means of that in the Fibonacci series we do addition of the two previous number where as I thought to be multiply the two previous number and find the $\mathrm{n}^{\text {th }}$ number from the series. Here, the in the multiplication series we can see that it get higher and higher at each terms and thus I say it as Highly Multiplicative Series. The all four conditions are applicable for all type of integers either it is bigger or smaller. eg:- $1,2,3 \ldots$ to 190000 etc.

\section{REFERENCES}

[1]. The Fibonacci Series [fibonaccinumbers.com] . 